\title{
5469 Detecting Sub-Seismically Stacked Fluvial Channel Sequences From Integration of Well-to-Seismic Data
}

K. de Leeuw (PDO) \& F. Neves (PDO)

The Upper and Middle Gharif units in North Oman comprise alternating fluvial channel and floodplain facies, with stacked fluvial sequences of up to 15 meters in thickness. These fluvial channels are prolific, producing reservoirs in North Oman and can be charged with both oil and gas. With decreasing channel connectivity (N/G) from the provenance area towards the Rub Al-Khali Basin in the NW, the greatest risk with this play is finding connected reservoir within a trap. Direct observations from seismic have proven to be unreliable, with stacked fluvial channels being below seismic resolution and the degree of lateral variation of rock physical properties of the various facies deposited within the Gharif. Seismic quality varies over the area as well, with the older vintages showing more seismic artifacts than newer acquisitions, impairing proper calibration of wells and quantitative correlation between surveys.

Neural Networks (NN) may prove useful to detect features that are below seismic wavelength provided that seismic quality is good, shot over an area with ample well control with rock physics showing some correlation with some seismic properties. To this end one of the best seismic survey in North Oman was chosen over a producing field with well constrained facies and fluid data.

First a seismic waveform classification was done over the Upper and Middle Gharif to assess variability of seismic facies and the correlation with the regional geological model. This showed that variation in seismic facies was parallel to inferred flow direction of the channel systems in this area. Second a NN training set was created over the Upper and Middle Gharif consisting of 9 wells with varying facies distributions both with varying N/G as well as similar N/G having net sand located at different layers in the model. As Gamma-Ray (GR) or Volume of Shale (V-shale) showed the best correlation with seismic properties, GR was predicted on the basis of seismic properties. To this end several seismic attribute volumes were generated including an acoustic impedance inversion cube to enhance the resolution (frequency bandwidth) of the seismic. Predicting GR using these volumes had an accuracy of up to $90 \%$.

Finally, the training set was used to create a GR over the seismic away from the wells, generating a 3D GR cube. The availability of many blind wells not used in the training set over this field allowed us to correlate unused wells to the GR-cube resulting in an accuracy of $75 \%$. This GR-cube shows strong indication of a high N/G area down-dip from the field that seems unconnected to the system that is present over the field. This shows the true potential of the method by unlocking additional STOIIP in known areas that might be stratigraphically (component) trapped. 